\title{
LOCALLY COMPACT SEMIGROUPS WITH DENSE MAXIMAL SUBGROUPS
}

BY

T. S. WU

1. Introduction. Let $S$ be a locally compact semigroup with a dense maximal subgroup $G$. We ask the following question: When is $G$ a topological group under the induced topology? It was proved recently by P. S. Mostert [8] that in general $G$ is not a topological group. However, there are several good reasons motivating the study of such a problem.

First, for an arbitrary locally compact semigroup, the closure of the maximal subgroup containing an idempotent forms a locally compact semigroup. If we know this maximal subgroup is a topological group, then it is a locally compact topological group. (This fact will be proved in the following paragraph.) By our rather good knowledge of locally compact topological groups, we can obtain information about the structure of this locally compact semigroup.

Second, if we look at this problem from the point of view of transformation groups, we have a group $G$ acting on a locally compact topological space. Naturally, we would like this group to be topologized so that it is a topological group. It is known that the weakest topology for $G$ to be a topological group is the $g$-topology [1]. It is somewhat harder to deal with the $g$-topology than with the $k$-topology, but under some conditions the $g$-topology will coincide with the $k$-topology. Among the known conditions, the local compactness of the group seems most interesting. If one tries to generalize such a condition, it is rather natural to ask the following: If $G$ is a group of homeomorphisms of $X$ onto $X$, and the closure of $G$ is locally compact in the $k$-topology, does the induced topology of $G$ coincide with the $g$-topology?

Third, in the past few years there have been several papers about such questions: What is the relationship between the algebraic structure and the topological structure? When can one strengthen the topological structure so that even under weak conditions the algebraic operations will be continuous in a certain sense? For instance, in topological groups, if the group space is locally compact, and if the multiplication is separately continuous with respect to the topology of the space, then the group is a topological group. We are able to prove the same theorem when the space is second countable and second category without the assumption of local compactness [9]. Now, our present problem arises quite

Received by the editors May 13, 1963. 
naturally along this line. In the following we will first state the general facts about this problem. Then we will try to give several conditions which will guarantee that the group is a topological group.

In $\$ 3$ we give a procedure of producing the locally compact semigroups with maximal dense groups such that the maximal dense subgroup is not a topological group under the induced topology. Such semigroups will be called a semigroup of Mostert type. In $\$ 4$ the proof of the following fact is given.

If $S$ is abelian and if the component containing the identity of $e$ is discrete, then either $G$ is a topological group or $S$ contains a subsemigroup of Mostert type.

In $\$ 5$ we discuss the case where $S$ or $G$ is connected, or locally connected. Mainly, we prove that if $G$ is connected and $S$ is second countable, then $S$ contains a subgroup which is the faithful representation of the additive group of reals.

$\S 6$ is about the case where the topology of $G$ is second category. In $\$ 7$ certain other aspects of our problem in this paper are discussed. We have included some results by Mostert [8] for completeness.

A portion of this paper is taken from Chapter 1 of the author's dissertation (Tulane University, 1962), which was written under the supervision of Professor P. S. Mostert, to whom I would like to express my gratitude. I would also like to express my thanks to Doctor A. L. Hudson who read this paper very carefully and made many valuable suggestions.

\section{Definitions and preliminaries.}

(2.1) Definition. A topological semigroup is a $W$-semigroup if and only if it is locally compact and has a maximal dense subgroup.

(2.2) Convention. In this chapter we shall always use $S$ to denote a $W$-semigroup, $G$ its maximal dense subgroup, and $e$ the identity of $G$.

(2.3) Definition [8]. We say a $W$-semigroup $S$ is tame (or $S$ is a tame semigroup) when its maximal dense subgroup is a topological group under the induced topology, otherwise $S$ is wild (or $S$ is a wild semigroup).

(2.4) Definition. Let $X$ be a locally compact Hausdorff space and let $C[X, X]$ be the semigroup of all continuous functions from $X$ to $X$. We shall use $C_{k}[X, X]$ to denote the topological space obtained from $C[X, X]$ by the compact open topology. If $A$ is a subset of $C_{k}[X, X]$, we say that $A$ is topologized by the $k$-topology.

(2.5) Definition. Two semigroups $T$ and $T^{\prime}$ are iseomorphic if there is a function $f$ from $T$ onto $T^{\prime}$ such that $f$ is a homeomorphism and an isomorphism.

(2.6) Proposition. If $T$ is a locally compact semigroup with unit $e$, then $T$ can be embedded into $C_{k}[T, T]$; i.e., $T$ is iseomorphic to a subsemigroup of $C_{k}[T, T]$. 
Proof. It is well known that $C_{k}[T, T]$ is a topological semigroup when we define the semigroup operation by the composition of functions. Define $\Phi: T \rightarrow C_{k}[T, T]$ by $\Phi(t)=f_{t} \in C_{k}[T, T]$, where $f_{t}(s)=t s$ for $t, s \in S$. Since, $f_{t}(e)=t, f_{s}(e)=s$, if $t \neq s, f_{t} \neq f_{s}$. Hence $\Phi$ is one-one.

To prove the continuity of $\Phi$, let $f_{t} \in[K, U]=\{f: f \in C[T, T], f(K) \subset U$, where $K$ is compact and $U$ is open $\}$, then $t K \subset U$. By the continuity of multiplication, there is an open neighborhood $V$ of $t$ such that $V K \subset U$, i.e., $f_{p}(K) \subset U$ and $f_{p} \in[K, U]$ if $p \in V$. In other words $\Phi(V)=\left[f_{p}: p \in V\right] \subset[K, U]$.

Now let $V$ be any open subset of $T$, since $\Phi(V)=\left[f_{v}: v \in V\right]=[e, V] \cap \Phi(T)$, $\Phi(V)$ is open in $\Phi(T)$. From above it follows that $\Phi$ is an iseomorphism.

(2.7) Definition [1]. Let $X$ be a locally compact space, and topologize $C[X, X]$ by taking $\{[K, U]: K$ is closed, $U$ is open and either $K$ or $X-U$ is compact $\}$ as subbasis for the topology. We shall denote this topological space by $C_{g}[X, X]$ and say that $C_{g}[X, X]$ is topologized by the $g$-topology.

(2.8) Remark. Let $S$ be a locally compact semigroup with a dense subgroup $G$. By (2.6) $S$ is iseomorphic to a subsemigroup of $C_{k}[S, S]$. Algebraically, $S$ is isomorphic to a subsemigroup of $C[S, S]$, hence it can be topologized by the $g$-topology. In order to distinguish this new topology from the $k$-topology, we shall use $G(g), S(g)$ to denote the topological spaces obtained by the $g$-topology. Actually, our main problem in this paper is to find conditions under which $G$ is iseomorphic to $G(g)$.

(2.9) Proposition. Let $g_{v}$ be a net of homeomorphisms of a locally compact space onto itself. If $g_{v}$ converges to a function $f$ under the $g$-topology, then $f$ is an onto map.

Proof. Obvious.

(2.10) Proposition. If $S$ is an abelian $W$-semigroup, then $G(g)$ is closed in $S(g)$.

Proof. Let $h_{v}$ be a net in $G(g)$ converging to $h$ in $S(g)$. Then by (2.9), $h$ has to be an onto map, i.e., $h S=S=S h$. Hence, there is an element $f \in S$ such that $f h=h f=e$; this implies $h \in G$, a fortiori, $G(g)$ is closed in $S(g)$.

(2.11) Proposition [1]. If $X$ is a locally compact space and $G$ is a group of homeomorphisms of $X$ onto itself, then the g-topology is the coarsest topology such that $G$ is a topological group.

(2.12) Proposition [1]. If $X$ is a locally compact, locally connected space, and $G$ is a subgroup of $C_{k}[X, X]$, then $G(k)$ is iseomorphic to $G(g)$, i.e., $G(k) \subset C_{k}[X, X]$ is a topological group.

(2.13) Lemma [2]. Let $V$ be a compact subset of a semigroup $S$. Then $V^{-1}=\left[v \in V \mid\right.$ if $v^{-1}$ exists $]$ is closed. 
(2.14) Lemma. Let $S$ be a W-semigroup. Then by taking $\left[V \cap V^{-1} ; V\right.$ is a neighborhood of $e$ ] as a fundamental neighborhood system, $G$ is a topological group, and this topology coincides with the g-topology if we embed the semigroup $S$ into $C_{\varepsilon}[S, S]$.

(2.15) Proposition. Suppose $S$ is a $W$-semigroup; then $S$ is tame if and only if $G$ is a locally compact subset of $S$.

Proof. 1. If $G$ is locally compact, then by a theorem of Ellis [3], $G$ is a topological group.

2. Suppose $S$ is tame. Let $V$ be a compact neighborhood of $e$ such that $V \cap G=V \cap V^{-1}$ is closed by (2.13). It is a compact subset of $V$ since $V \cap V^{-1} \subset G$, it is also compact in $G$, hence $G$ is locally compact.

(2.16) Corollary. Let $S$ be a $W$-semigroup. Then $S$ is tame if and only if $G$ is open in $S$.

Proof. A locally compact dense subset in a Hausdorff space is open. Conversely, an open subset of a locally compact Hausdorff space is locally compact.

(2.17) Corollary. Suppose $S$ is a tame $W$-semigroup. Then $G$ is second category in itself.

Proof. Since $G$ is locally compact, it is second category by the Baire theorem.

(2.18) Corollary. Suppose $S$ is a tame $W$-semigroup. Then $G$ is fully normal.

Proof. A locally compact topological group is fully normal.

(2.19) Proposition. Let $H$ be a subgroup of a $W$-semigroup $S$, and $H^{*}$ be the closure of $H$ in $S$. Then $H^{*}$ is a $W$-semigroup, and $H^{*}$ is tame if and only if $H$ is a topological group under the induced topology.

Proof. It is clear that $H^{*}$ is a locally compact semigroup. If $H^{*}$ is tame, i.e., the maximal dense subgroup is a topological group, then $H$, as a subgroup of a topological group, is a topological group. The proof of the converse can be found in a lemma of Hofmann's [4].

(2.20) Proposition. Suppose $S$ is a $W$-semigroup. If $S$ has a $G$-invariant uniformity, then $S$ is tame.

(2.21) Proposition. Every $W$-semigroup has a maximal compact connected subgroup.

Proof. If we topologize $G$ by the $g$-topology, then $G(g)$ is a locally compact topological group. Let $K(g)$ be the identity component of $G(g)$. Then $K(g)$ contains a maximal compact connected subgroup $C(g)$ by the structure theorem of locally compact connected topological groups [5]. Let $i$ be the inclusion map from $G(g)$ to $G$. Then $i$ is continuous, hence $i(C(g)) \subset G$ is iseomorphic to $C(g)$. If $H$ is a compact connected subgroup, since $H$ is compact, it is a topological 
group. Hence $H$ is iseomorphic to $H(g)$. And therefore $H(g) \subset K(g)$, and $H(g)$ is contained in some maximal compact connected subgroup which is conjugate to $C(g)$. Hence $C(g)$ is maximal in $C$, i.e., $C(k)=i(C(g))$ is a maximal compact connected subgroup.

(2.22) Proposition. Let $S$ be an abelian $W$-semigroup, and $R=[(a, b): a K$ $\cap b K \neq \Phi$ where $K$ is the maximal compact connected subgroup of $S]$. Then $R$ is an equivalence relation and $S^{\prime}=S / R$ is a locally compact $W$-semigroup when $S^{\prime}$ is topologized by quotient topology and the multiplication in $S^{\prime}$ is defined by $a K * b K=a b K$.

Proof. It is clear that if $a K \cap b K \neq \Phi$, then $a K=b K$, and $R$ is an equivalence relation. Also, it is clear that $S^{\prime}$ is locally compact. Let $a, b$ be two arbitrary elements in $S$, and $\theta(a b) \in V \subset S^{\prime}$, where $\theta$ is the canonical map and $V$ is open in $S^{\prime}$. Then $\theta^{-1}(V)=\theta^{-1}(V) K$ is open in $S, a b K \subset \theta^{-1}(V) K$, hence there are open sets $V_{1}, V_{2}$, containing $a, b$ respectively, such that $V_{1} K V_{2} K=V_{1} V_{2} K$ $\subset \theta^{-1}(V), K$ i.e., $\theta\left(V_{1}\right) * \theta\left(V_{2}\right) \subset V$. Thus $S^{\prime}$ is a locally compact semigroup.

3. Construction of wild semigroups. In this section we shall give a procedure for producing wild semigroups.

(3.1) Definition (Mostert product [8]). Let $\left(E_{i}, F_{i}, I\right)$ be a collection where $I$ is an infinite set for which $i \in I, E_{i}$ is a topological space, and $F_{i}$ a subspace of $E_{i}$. Define $S$ to be the collection of all functions $f: I \rightarrow \bigcup_{i} E_{i}$ such that $f(i) \in E_{i}$, and $f(i) \in F_{i}$ except for finitely many values of $i$. Take for a subbasis for a topology on $S$ the following sets: for a finite subset $N$ of $I, U_{i}$ open subset of $E_{i}(i \in N)$, let $U_{N}=\left[f \in S: f(i) \in U_{i}(i \in N)\right.$ and $\left.f(j) \in F_{j}(j \notin N)\right]$. Thus $F=\prod_{i} F_{i}$ is an open subset of $S$ on which the topology agrees with Tychonoff topology.

(3.2) Proposition. If each $E_{i}$ is a locally compact topological space and $F_{i}$ is a compact subset of $E_{i}$ for $i \in I$, then the Mostert product $S$ of $\left(E_{i}, F_{i}, I\right)$ is locally compact.

Proof. Let $f \in S$. If $f \in \prod_{i} F_{i}$, then $\prod_{i} F_{i}$ is a compact open neighborhood for $f$. If $f(i) \in F_{i}$ except for a finitely many index subset $N$ of $I$, since $F_{i}$ is a compact subset of $E_{i}$ for $i \in N$, there is a compact neighborhood $U$ of $f(i)$ such that $U_{i} \cap F_{i}=\square$, then $\prod_{i \in N} U_{i} \times \prod_{i \in I-N} F_{i}$ is the compact neighborhood of $f$.

(3.3) Proposition. Let $H$ be a compact monothetic semigroup with generator $b$ and identity $u$. Then there exists $a W$-semigroup $E$ with the following properties:

(i) The maximal dense subgroup $G$ of $E$ is infinite cyclic.

(ii) $G$ is discrete under the relative (induced) topology.

(iii) $E-G$ is isomorphic to $H$, so we shall identify $E-G$ with $H$.

(iv) Let $g \in G$ be the generator. Then $g u=b$.

Proof. Let $R$ be the semigroup formed by non-negative reals under usual multiplication and topology. Let $X=H \times R$ be the semigroup formed by the 
cartesian product of $H$ and $R$. Then $E=\left[\left(b^{m}, 2^{-m}\right) \mid-\infty<m<\infty\right] \cup[(h, 0) \mid$ $h \in H]$ as a subsemigroup of $X$ is what we claimed in the proposition. $E$ is a closed subset of $X$. If a sequence $\left\{\left(x_{n}, y_{n}\right)\right\}$ in $E$ converges to $(x, y) \in X$, then $x_{n}$ converges to $x$ and $y_{n}$ converges to $y$. But the only limiting point of the set $\left\{2^{m} \mid-\infty \leqq m<\infty\right\}$ is 0 . Hence $(x, y) \in H \times\{0\}$, which is closed. So $E$ is a closed subset of $X, E$ is locally compact. It is clear that $\left(b^{0}, 1\right)=(u, 1)$ is the identity of $E$ and the maximal subgroup $G$ of $E$ is the subset $\left[\left(b^{m}, 2^{-m}\right) \mid-\infty<m<\infty\right]$ and $G$ is infinite cyclic with generator $g=\left(b, 2^{-1}\right)$. Since $G$ is the complement of the compact subset $H \times\{0\}$ in $E$, it is open, hence locally compact and countable, so $G$ is discrete under the induced topology. To see that $G$ is dense in $E$, let $U \times V$ be a neighborhood of $(h, 0)$ where $h \in H$. Since $H$ is a monothetic group with generator $b, h=\lim b^{m_{i}}$, where $m_{i}$ is a monotonic increasing sequence of integers [10], hence we can find a sufficient large $m$ such that $b^{m} \in V$ and $2^{-m} \in V$, i.e., $\left(b^{m}, 2^{-m}\right) \in U \times V$.

(iii) is clear.

To prove (iv), $g=\left(b, 2^{-1}\right), g u=\left(b, 2^{-1}\right)(u, 0)=(b u, 0)=(b, 0)$.

(3.4) EXAMPLes.

(3.4a) Let $H$ consist of only one element $\{u\}$. Then $E$ is iseomorphic to $\left[2^{m} \mid-\infty \leqq m<\infty\right]$.

(3.4b) Let $H$ be the circle group or Cantor dyadic group. Then $E$ can be visualized in Euclidean three space.

(3.5) Notation. Let $S$ be a semigroup with identity $e$. Let $a \in S$. Let $\Delta(a)=\left[a^{n} \mid n \geqq 0\right]^{*}$, the closure of the set of positive powers of $a$ in $S$, where $a^{0}=e$.

(3.6) Proposition. Let I be an infinite set, for each $i \in I$. Let $E_{i}$ be a $W$-semigroup constructed as in (3.3) with the generator $g_{i}=\left(b_{i}, 2^{-1}\right)$ for the maximal dense subgroup $G_{i}$. Let $F_{i}=\Delta\left(g_{i}\right)$. Then the Mostert product $S$ of $\left(E_{i}, Z_{i}, I\right)$ is a wild $W$-semigroup where the semigroup operation is the coordinative multiplication.

Proof. (i) $F_{i}=\Delta\left(g_{i}\right)$ is compact. Since $\Delta\left(g_{i}\right)=H_{i} \times\{0\} \cup\left[g_{i}^{n} \mid g_{i}=\left(b_{i}, 2^{-1}\right)\right.$, $n \geqq 0]$ is a closed subset of the compact set $H \times[0,1]$, so $S$ is locally compact by (3.2).

(ii) We shall prove that $S$ is a topological semigroup. Let $f, g \in S$. We note that $\prod_{k \in K} F_{k}$ is a semigroup under coordinatewise multiplication, $K \subset I$. Let $Z=\prod_{j \in J} Z_{j} \times \prod_{k \in I-J} F_{k}$ be a neighborhood of $f g$ where $J$ is a finite subset of $I$, and $Z_{j}$ is an open subset of $E_{j}$. Since $E_{j}$ is a topological semigroup there are neighborhoods $U_{j}, V_{j}$ of $f(j), g(j)$ respectively such that $f(j) g(j) \in U_{j} \cdot V_{j} \subset Z_{j}$. Then it is clear that

$$
f g \in\left\{\prod_{j \in J} U_{j} \times \prod_{k \in I-J} F_{k}\right\}\left\{\prod_{j \in J} V_{j} \times \prod_{k \in I-J} F_{k}\right\} \subset Z .
$$


(iii) The maximal dense subgroup $G$ of $S$ is the set $\left[g \mid g(i) \in G_{i}, g(i)=e_{i}\right.$ except finitely many $i \in I]$. It is not a topological group under induced topology. Since $g_{n} \in G$ where $g_{n}(n)=\left(b_{n}, 2^{-1}\right) \in G_{n}$, and $g_{n}(m)=e_{m}$ is a sequence in $G$ converging to the identity of $G, g_{n}^{-1} \notin \prod_{i \in I} F_{i}$, hence $G$ is not a topological group.

(3.7) Remark. As we mentioned at the beginning of this paper, P. S. Mostert had been the first one to construct the wild semigroup. His example is formed by the Mostert product of $\left(E_{i}, F_{i}, I\right)$ where $E_{i}=\left\{2^{m} \mid-\infty \leqq m<\infty\right\}$, $F_{i}=\left\{2^{m} \mid-\infty \leqq m \leqq 0\right\}$ (cf. (3.4a)).

(3.8) Definition (Semigroup of Mostert tyPe). The wild semigroups which can be obtained by (3.6) are called semigroups of Mostert type.

(3.9) Remark. It is clear that we can obtain further examples by using the cartesian product of wild semigroups with either $W$-semigroups or locally compact topological groups.

4. A structure theorem for wild semigroups. In this section we shall prove that if the wild semigroup is abelian second countable and also the component containing $e$ is degenerate, then this semigroup contains a subsemigroup of Mostert type. The proof is divided into several lemmas. First we need the following lemma.

(4.1) LEMMA [7]. Let $S$ be a locally compact semigroup with identity e, and suppose the component $C$ containing $e$ is compact. Then any open set about $C$ contains an open compact semigroup $K$ about $C$.

(4.2) CONVENTION FOR THIS SECTION ONLY. For simplicity we made the following convention for this section: $S$ is a locally compact second countable wild abelian $W$-semigroup whose component containing $e$ is degenerate. The maximal dense subgroup of $S$ is denoted by $G$, identity element $e$.

(4.3) Lemma. There is a sequence $\left\{g_{n} \mid n \in \omega\right\}$ in $G$ such that

(i) $g_{n}$ converges to $e$,

(ii) $g_{n}^{-1}$ does not have any convergent subsequences,

(iii) there exists a compact open subsemigroup $K$ such that $e \in K$, $g_{n} \in K, g_{n}^{-1} \notin K$ for all $n \in \omega$.

Proof. Since $S$ is wild there is a sequence $\left\{f_{n}\right\} \in G$ such that $\left\{f_{n}\right\}$ converges to $e$ and $f_{n}^{-1}$ does not converge to $e$. We can select $\left\{f_{n}\right\}$ in such a way that $\left\{f_{n}^{-1}\right\}$ does not have convergent subsequences. Since $\left\{f_{n}\right\}$ converges to $e$, and assuming the subsequence $\left\{f_{n_{i}}^{-1}\right\}$ converges to $s \in S$, then $\lim f_{n_{i}} \lim f_{n_{i}}^{-1}$ $=\lim f_{n_{i}} f_{n_{i}}^{-1}=e$. On the other hand, $\lim f_{n_{i}} \lim f_{n_{i}}^{-1}=e s=s$. This implies that $s=e$. If every subsequence of $\left\{f_{n}^{-1}\right\}$ is convergent, then $\left\{f_{n}^{-1}\right\}$ will converge to $e$ too. This gives the contradiction. Since $\left\{f_{n}^{-1}\right\}$ does not converge to $e$, by (4.1), we can find an open compact subsemigroup $K$ containing $e$ and all $f_{n} \in K$ 
except possibly finite number elements. We reindex those $f_{n}$ which belong to $K$ and denote it by $g_{n}$. Then $\left\{g_{n}\right\}$ satisfies (i), (ii) and (iii).

(4.4) LEMMA. There exists a wild $W$-subsemigroup $T$ of $S$ such that $T$ is the closure of a free abelian subgroup $H$ generated by countable many elements $\left\{h_{n} \mid n \in \omega\right\} \subset G$.

Proof. The existence of $h_{n}$ is proved by induction. Let $K$ and $g_{n}$ have the same meaning as in (4.3). Let $h_{1}=g_{1}$ and $K_{1}=K$. Assume $h_{i}$ and $K_{i}$ have been defined for $i \leqq m$ and have the following properties: (i) $h_{i} \in\left\{g_{n} \mid n \in \omega\right\}$, (ii) $K_{i}$ is an open compact subsemigroup of $S$ and $K_{i} \subset K_{i-1}$, (iii) $e \in K_{i}$, (iv) $h_{i} K_{i}$ $\cap K_{i+1}=\square$. Since $g_{n}^{-1} \notin K_{1}=K, e \notin h_{m} K_{m}$, by (4.1), there is an open compact subsemigroup $K_{m+1}$ of $K_{m} l$ such that $e \in K_{m+1}$ and $h_{m} K_{m} \cap K_{m+1}=\square$. As $\left\{g_{n} \mid n \in \omega\right\}$ converges to $e$, there is a $g_{l} \in K_{m+1}$. Let $h_{m+1}=g_{l}$. It is clear that $h_{i}$ and $K_{i}$ satisfy (i)-(iv) for $1 \leqq i \leqq m+1$.

We are going to prove that $\left\{h_{n} \mid n \in \omega\right\}$ is linearly independent, i.e., if $h_{s_{1}}^{r_{1}} \cdots h_{s_{n}}^{r_{n}}=e, s_{1}<s_{2}<\cdots<s_{n}$, then $r_{1}=r_{2}=\cdots=r_{n}=0$. Suppose $h_{s_{1}}^{r_{1}} \cdots h_{s_{n}}^{r_{n}}=e, s_{1}<s_{2}<\cdots<s_{n}$, and $0<r_{i}$. Then $h_{s_{1}}^{r_{1}} \cdots h_{s_{n}}^{r_{n}} \in h_{s_{1}}^{r_{1}} K_{s_{1}} \subset h_{s_{1}} K_{s_{1}}$. But $e \in K_{s+1}$ and $K_{s_{1}+1} \cap h_{s_{1}} K_{s_{1}}=\square$. This gives us the contradiction. Suppose $h_{s_{1}}^{r_{1}} h_{s_{2}}^{r_{2}} \cdots h_{s_{n}}^{r_{n}}=h_{t_{1}}^{p_{1}} \cdots h_{t_{m}}^{p_{m}}$, where $s_{1}<s_{2}<\cdots<s_{n}, 0<r_{i}, t_{1}<t_{2}<\cdots<t_{m}, 0<p_{i}$. Then $h_{s_{1}}^{r_{1}} \cdots h_{s_{11}}^{r_{n}} \in h_{s_{1}} K_{s_{1}}, h_{t_{1}}^{p_{1}} \ldots h_{t_{m}}^{p_{m_{m}}} \in h_{t_{1}} K_{t_{1}}$. Without loss of generality we can assume $s_{1}<t_{1}$. Then $h_{s_{1}} K_{s_{1}} \cap K_{s_{1}+1}=\square$ and $K_{t_{1}} \subset K_{s_{1}+1}, h_{t_{1}} K_{t_{1}} \subset K_{t_{1}} K_{t_{1}} \subset K_{t_{1}}$ $\subset K_{s_{1}+1}$. This also gives us the contradiction.

Now let $H$ be the subgroup generated by $\left\{h_{n} \mid n \in \omega\right\}$, and $T$ the closure of $H$. Then $T$ is a locally compact semigroup. It is wild since $\left\{h_{n}\right\}$ is a subsequence of $\left\{g_{n}\right\}$ converging to $e$ but $h_{n}^{-1}$ does not have any convergent subsequence.

(4.5) Standing notations. From now on, in this section, we shall use $T$ to denote the subsemigroup defined in (4.4). $T$ is the closure of the free group generated by $\left\{h_{n} \mid n \in \omega\right\}$. We shall use $h$ to denote any one of these generators $\{h \mid n \in \omega\}$. Let $s \in S$. The following notations are standard [7].

$$
\begin{aligned}
& \Gamma_{n}(s)=\left\{s^{i} \mid i \geqq n\right\}^{*} . \\
& \Gamma(s)=\Gamma_{1}(s) . \\
& K(s)=\bigcap\left\{\Gamma_{n}(s) \mid n \in \omega\right\} . \\
& O(s)=\left\{s^{i} \mid i \geqq 1\right\} .
\end{aligned}
$$

Let $g \in G \subset S$, and $A(g)$ denotes $O(g) \cup O\left(g^{-1}\right) \cup\{e\}$. It is clear that $A(g)$ is a group and $A(g)^{*} \neq \Gamma(g) \cup \Gamma\left(g^{-1}\right) \cup\{e\}$ is a subsemigroup.

(4.6) LeMma. If $\Gamma(s)$ is compact, then $K(s)$ is the minimal ideal of $\Gamma(s)$ as well as the unique maximal subgroup of $\Gamma(s)$.

$$
\Gamma(s)=K(s) \cup\{O(s)-K(s)\}
$$

and $O(s)-K(s)$ is a discrete open subset of $\Gamma(s)$.

Proof. This lemma is well known; for reference of the proof see [7]. 
(4.7) LEMMA. $\Gamma\left(h_{n}\right)$ is a compact monothetic semigroup where $h_{n}$ is defined in (4.4).

Proof. We use $h$ to denote $h_{n}$ for any fixed $n \in \omega$. From (4.4) $h^{-1} \notin K$, $e \notin h K, e \notin \Gamma(h)$. Since $\Gamma(h)$ is compact and does not contain $e, O(h)$ is not dense in itself. So we have $O(h) \cap K(h)=\square$. By (4.6) $\Gamma(h)$ is the disjoint union of the maximal subgroup $K(h)$ and $O(h)$. Let $u$ be the identity element of $K(h)$. Let $s \in K(h), s=\lim h^{n_{i}}$ for some sequence of positiveintegers $n_{i}$. So $s=s u=\lim h^{n_{i}} u$, $h^{n_{i}} u \in K(h)$. Hence $K(h)$ is a compact monothetic group with generator $h u$ and identity $u$.

(4.8) Lemma. $A(h) \cup K(h)$ is a locally compact subsemigroup of T. $A(h)$ is a discrete topological group when it is equipped by relative topology of $T$.

Proof. Since $\Gamma(h) \cup\{e\}$ is compact, $A(h) \cup K(h)=\{\Gamma(h) \cup\{e\}\} \cup\{\Gamma(h) \cup\{e\}\}^{-1}$. As the union of two closed sets, it is a closed subset of $T$. Hence $A(h) \cup K(h)$ is locally compact. It is easy to see that $A(h) \cup K(h)=A(h)^{*}$, so $A(h) \cup K(h)$ is a locally compact semigroup. Since $K(h)$ is compact, $A(h)$ is a countable, locally compact topological group; $A(h)$ is discrete under the relative topology.

(4.9) Remark. For realization of $A(h) \cup K(h)$, one may refer to (3.3). The proof of this fact follows from a theorem by Hewitt [11] and (4.7), (4.8).

(4.10) REMARK AND NOTATIONS. We shall sketch the procedure of our proof that $S$ has a subsemigroup of Mostert type (4.20). Let $E_{i}=A\left(h_{i}\right) \cup K\left(h_{i}\right)$, $F_{i}=\Gamma\left(h_{i}\right) \cup\left\{e_{i}\right\}, e_{i}$ the identity of $E_{i}$. Let $X$ be the semigroup of Mostert type formed by $\left(E_{i}, F_{i}, i \in \omega\right)$. It is clear that $Y=\left\{f \in X \mid f(i) \in O\left(h_{i}\right) \cup\left\{e_{i}\right\}\right.$ and $f(i)=e_{i}$ for all $i$ except possibly finite indices $\}$ is a subsemigroup of $X$. Let $Z=Y^{*}$. From the structure of $X, X=Z Z^{-1}$ and $Z$ is an open compact subsemigroup containing the identity of $X$. Also we know that the topology of $Z$ is equivalent to the product topology of $\prod_{i \in \omega}\left[\Gamma\left(h_{i}\right) \cup\left\{e_{i}\right\}\right]$. We shall single out subsets $F, P, Q$ of $T$ corresponding to $Y, Z, X$ respectively. Let $F$ be the subsemigroup of $T$ generated by $\left\{h_{i} \mid i \in \omega\right\} \cup\{e\}$. Let $P=F^{*}$ and $Q=P P^{-1}$. We shall first prove that $F$ and $Y$ are uniformly iseomorphic (Lemmas 4.11-4.15). Then we shall prove that $P$ is an open subset of $Q$ and $Q$ is a locally compact semigroup (Lemmas 4.16-4.18). The last step (4.19) is to prove that $Q$ is iseomorphic to $X$, a fortiori, $S$ has a subsemigroup of Mostert type.

(4.11) LeMma. $P$ is a compact semigroup. If $\varepsilon>0$, an arbitrary positive number, and $m$ a fixed positive integer, then there is a positive number $\delta>0$ such that if $d\left(h_{i}^{s}, h_{i}^{t_{i}}\right)<\delta$ for $i=1,2, \cdots, m$, then $d\left(h_{1}^{s_{1}} \cdots h_{m}^{s_{m}}, h_{1}^{t_{1}} \cdots h_{m}^{t_{m}}\right)<\varepsilon$ where $d$ is a metric defined on $P$ compatible with the unique uniformity on $P$.

Proof. Since $\left\{h_{i} \mid i \in \omega\right\}$ is contained in the compact subsemigroup $K$ of $T$, hence $P=F^{*}$ is a compact semigroup. The second statement of the lemma follows from the uniform continuity of the semigroup multiplication on $K_{1}$. 
(4.12) LEMMA. There is a natural algebraic isomorphism $\rho$ from $F$ onto $W$.

Proof. Let $h_{s_{1}}^{r_{1}} \cdots h_{s_{n}}^{r_{n}} \in F$. Define $\rho\left(h_{s_{1}}^{r_{1}} \cdots h_{s_{n}}^{r_{n}}\right)=f$ where $f\left(s_{i}\right)=h_{s_{i}}^{r i}, f(j)=e_{j}$ if $j \neq s_{i}$ for $i=1, \cdots, n$. It is clear that $Y$ is iseomorphic to the (semigroup) weak direct sum $A$ of $\left\{O\left(h_{i}\right), e_{i} \mid i \in \omega\right\}$. Since $\left\{h_{i} \mid i \in \omega\right\}$ is linearly independent, $F$ is iseomorphic to $A$. The function $\rho$ gives the iseomorphism.

(4.13) LEMMA. $\rho$ is uniformly continuous with respect to the product uniformity of $Y$ and the relative uniformity of $P$ on $F$.

Proof. Obvious.

(4.14) LEMMA. $\rho^{-1}$ is uniformly continuous.

Proof. Let $d$ be the metric defined in (4.11). Define the metric $\tilde{d}$ on $Z$ by $\tilde{d}\left(f, f^{\prime}\right)=\sum_{n \in \omega} 2^{-n} d\left(f(n), f^{\prime}(n)\right)$. It is well known that $\tilde{d}$ is compatible with the product uniformity on $Z$. Since $P$ is compact, the semigroup action of $P$ on $P$ is equicontinuous, i.e, $P \subset C_{k}[P, P]$, a fortiori, $F$ is equicontinuous. Let $\varepsilon>0$. There is a neighborhood $U$ of $e$ such that $d(h e, h U)=d(h, h U)<\varepsilon / 3$ for $h \in F$. Since $U_{e}$ is a neighborhocd of $e$, there is an integer $m$ such that $K_{m} \cap F \subset U_{e}$ (cf. Lemma 4.4); so $d\left(h, h h_{s_{1}}^{r_{1}} \cdots h_{s_{n}}^{r_{n}}\right)<\varepsilon / 3$, when $s_{i} \geqq m, r_{i} \geqq 0$. By (4.11) there is a $\delta^{\prime}>0$ such that $d\left(h_{s_{1}}^{r_{1}} \cdots h_{s_{m}}^{r_{m}}, h_{s_{1}}^{t_{1}} \cdots h_{s_{m}}^{t_{m}}\right)<\varepsilon / 3$ when $d\left(h_{s_{i}}^{r_{i}}, h_{s_{i}}^{t_{i}}\right)<\delta^{\prime}$. Let $\delta=\delta^{\prime} / 2^{m+1}$. Suppose $f, f^{\prime}$ are elements in $Y, \rho^{-1}(f)=h_{s_{1}}^{r_{1}} \cdots h_{s_{n}}^{r_{n}}, \rho^{-1}\left(f^{\prime}\right)$ $=h_{s_{1}}^{t_{1}} \cdots h_{s_{n}}^{t_{n}}, s_{1}<s_{2}<\cdots<s_{n}$. If $\tilde{d}\left(f, f^{\prime}\right)=\sum 2^{-i} d\left(f(i), f^{\prime}(i)\right)<\delta^{\prime} / 2^{m+1}=\delta$, then $d\left(h_{s_{i}}^{r_{i}}, h_{s_{i}}^{t_{i}}\right)<\delta^{\prime}$ for $s_{i} \leqq m$. Let $S_{m}=$ Maximum $\left\{s_{i} \mid s_{i} \leqq m, i=1,2, \cdots, n\right\}$. By (4.11) $d\left(h_{s_{1}}^{r_{1}} \cdots h_{s_{m}}^{r_{m}}, h_{s_{1}}^{t_{1}} \cdots h_{s_{n}}^{t_{m}}\right)<\varepsilon / 3$. Since

$$
d\left(h_{s_{1}}^{r_{1}} \cdots h_{s_{m}}^{r_{m}}, h_{s_{1}}^{r_{1}} \cdots h_{s_{m}}^{r_{m}} U_{e}\right)<\varepsilon / 3, \quad d\left(h_{s_{1}}^{t_{1}} \cdots h_{s_{m}}^{t_{m}}, h_{s_{1}}^{t_{1}} \cdots h_{s_{m}}^{t_{m}} U_{e}\right)<\varepsilon / 3,
$$

so $d\left(h_{s_{1}}^{r_{1}} \cdots h_{s_{m}}^{r_{m}} U_{e}, h_{s_{1}}^{t_{1}} \cdots h_{s_{m}}^{t_{m}} U_{e}\right)<\varepsilon, d\left(h_{s_{1}}^{r_{1}} \cdots h_{s_{n}}^{r_{n}}, h_{s_{1}}^{t_{1}} \cdots h_{s_{n}}^{t}\right)<\varepsilon$. This proves that $d\left(\rho^{-1}(f), \rho^{-1}\left(f^{\prime}\right)\right)<\varepsilon$ when $\tilde{d}\left(f, f^{\prime}\right)<\delta$, so $\rho^{-1}$ is uniformly continuous.

(4.15) LeMma. $P$ is iseomorphic to $Z$.

Proof. From (4.14), we know that $\rho$ is a uniform iseomorphism. Hence the completions of (precompact) $Y$ and $F$ are homeomorphic, i.e., $Z$ and $P$ are homeomorphic. The iseomorphism of $P$ and $Z$ follows from the fact that $W$ is dense in $Y$ and $F$ is dense in $P$. We shall also use $\rho$ to denote this iseomorphism.

(4.16) LEMMA. $h_{s_{1}}^{-r_{1}} \cdots h_{s_{n}}^{-r_{n}}, h_{t_{1}}^{p_{1}} \cdots h_{t_{m}}^{p_{m}}$ does not belong to the compact subsemigroup $K$ of $T$, when $0<r_{i}, 0<p_{j}, 0<s_{1}<s_{2}<\cdots<s_{n}, 0<t_{1}<t_{2}<\cdots<t_{m}$, and $s_{i} \neq t_{j}$ for all $i, j$.

Proof. Suppose $h=h_{s_{1}}^{-r_{1}} \cdots h_{s_{n}}^{-r_{n}}, h_{t_{1}}^{p_{1}} \cdots h_{t_{m}}^{p_{m}} \in K$, then $\Gamma(h)$ is a compact subsemigroup. There is a sequence $\left\{h^{n_{i}} \mid n_{i}>0\right\}$ converging to some element $a$ in $K$. Also there exists a subsequence $\left\{m_{i} \mid i \in \omega\right\}$ of $\left\{n_{i}\right\}$ such that $\left\{h_{s_{i}}^{r_{i} m_{i}}\right\}$ converges to $a_{s_{i}} \in K\left(h_{s_{i}}\right)$ and $\left\{h_{t_{i}}^{p_{1} m_{i}}\right\}$ converges to $b_{t_{t}} \in K\left(h_{t_{1}}\right)$, for $i=1,2, \cdots, n$, $l=1, \cdots, m$.

Now 


$$
\begin{aligned}
\lim h^{m_{i}} \lim \left(h_{s_{1}}^{r_{1}} \cdots h_{s_{n}}^{r_{n}}\right)^{m_{i}} & =a a_{s_{1}} \cdots a_{s .} \\
& =\lim \left(h_{s_{1}}^{-r_{1}} \cdots h_{s .}^{-r_{n}}\right)^{m_{i}}\left(h_{t_{1}}^{p_{1}} \cdots h_{t_{m}}^{p_{m}}\right)^{m_{i}}\left(h_{s_{i}}^{r_{i}} \cdots h_{s_{n}}^{r_{n}}\right)^{m_{i}} \\
& =\lim \left(h_{t_{1}}^{p_{1}} \cdots h_{t_{m}}^{p_{m}}\right)^{m_{i}}=b_{t_{1}} \cdots b_{t_{m}} .
\end{aligned}
$$

On the other hand, let $u_{i}$ be the identity of $K\left(h_{i}\right)$; then

$$
\begin{aligned}
\left(\lim h^{m_{i}}\right) u_{s_{1}} \cdots u_{s_{n}} & =a u_{s_{1}} \cdots u_{s} \\
& =\lim \left(h_{s_{1}}^{-r_{1}} u_{s_{1}}\right)^{m_{i}} \cdots\left(h_{s_{n}}^{-r_{n}} u_{s_{n}}\right)^{m_{i}}\left(h_{t_{1}}^{p_{1}}\right)^{m_{i}} \cdots\left(h_{t_{m}}^{p_{m}}\right)^{m_{i}} \\
& =a_{s_{1}}^{-1} \cdots a_{s_{n}}^{-1} b_{t_{1}} \cdots b_{t_{m}} .
\end{aligned}
$$

Here, $a_{i} \in K\left(h_{i}\right), K\left(h_{i}\right)$ is a group, $a_{i}^{-1}$ is the inverse of $a_{i}$ in $K\left(h_{i}\right)$. Combining (1) and (2) we have $b_{t_{1}} \cdots b_{t_{m}}=u_{s_{1}} \cdots u_{s_{n}} b_{t_{1}} \cdots b_{t_{m}}$. This would imply $\rho\left(b_{t_{1}} \cdots b_{t_{m}}\right)=\rho\left(u_{s_{1}} \cdots u_{s_{n}} b_{t_{1}} \cdots b_{t_{m}}\right) \in Z$. But from the semigroup structure of $Z$, it is clear that this is not true in $Z$. So we have the contradiction and the proof is complete.

(4.17) Lemma. $Q=P P^{-1}$ is a locally compact subsemigroup of $T$.

Proof. It is clear that $P P^{-1}$ is a semigroup. We need to prove that $P P^{-1}$ is locally compact. By (4.16) $K$ does not contain any $h$ of the form described in (4.16). Also $P^{-1}=F^{-1}$ meets $K$ only at $\{e\}$. So $K \cap P P^{-1}=P$. This shows that $P$ is a compact open neighborhood of $e$ in $P P^{-1}$. Let $p h^{-1} \in P P^{-1}$, where $p \in P, h \in F$. Then $p h^{-1} P$ is a compact open neighborhood of $p h^{-1}$. Hence we know that $P P^{-1}$ is a locally compact subsemigroup of $T$.

(4.18) LEMMA. There is a natural algebraic iseomorphism from $Q$ onto $X$.

Proof. Let $p h^{-1} \in Q=P P^{-1}$. Define $\Phi\left(p h^{-1}\right)=\rho(p) \rho(h)^{-1}$. We need to prove that $\Phi$ is an algebraic semigroup isomorphism.

1. $\Phi$ is well defined: If $p h^{-1}=p^{\prime} h^{\prime-1}$, then $p h^{\prime}=p^{\prime} h, \rho\left(p h^{\prime}\right)=\rho\left(p^{\prime} h\right)$, $\rho(p) \rho\left(h^{\prime}\right)=\rho\left(p^{\prime}\right) \rho(h), \Phi\left(p h^{-1}\right)=\rho(p) \rho(h)^{-1}=\rho\left(p^{\prime}\right) \rho\left(h^{\prime}\right)^{-1}=\Phi\left(p^{\prime} h^{\prime-1}\right)$.

2. $\Phi$ is one-one: If $\Phi\left(p h^{-1}\right)=\Phi\left(p^{\prime} h^{\prime-1}\right)$, then $\rho(p) \rho(h)^{-1}=\rho\left(p^{\prime}\right) \rho\left(h^{\prime}\right)^{-1}$, $\rho(p) \rho\left(h^{\prime}\right)=\rho\left(p^{\prime}\right) \rho(h)$. Since $\rho$ is an isomorphism $\rho\left(p j^{\prime}\right)=\rho\left(p^{\prime} h\right), p h^{\prime}=p^{\prime} h$. Hence $p h^{-1}=p h^{-1}$.

3. $\Phi$ is a homomorphism: $\Phi\left(p h^{-1} p^{\prime} h^{\prime-1}\right)=\Phi\left(p p^{\prime}\left(h h^{\prime}\right)^{-1}\right)=\rho\left(p p^{\prime}\right) \rho\left(h h^{\prime}\right)^{-1}$ $=\rho(p) \rho\left(p^{\prime}\right)\left[\rho(h) \rho\left(h^{\prime}\right)\right]^{-1}=\Phi\left(p h^{-1}\right) \Phi\left(p^{\prime} h^{\prime-1}\right)$.

4. $\Phi$ is onto: Let $x f^{-1} \in X=Y Y^{-1}$. Then $\Phi\left(\rho^{-1}(x)\left[\rho^{-1}(f)\right]^{-1}\right)$ $=\rho \rho^{-1}(x)\left[\rho \rho^{-1}(f)\right]^{-1}=x f^{-1}$.

(4.19) Lemma. $\Phi$ is a homeomorphism.

Proof. 1. $\Phi$ is continuous: Since $P$ is a neighborhood of $e, \Phi|P=\rho| P$, $\rho$ is a homeomorphism. Hence $\Phi$ is continuous at every element in $P$. Let $p h^{-1} \in P P^{-1}$. From (4.17), $p h^{-1} P$ is a compact open neighborhood of $p h^{-1}$, 
$\Phi\left(P h^{-1}\right)=\rho(P) \rho(h)^{-1}$. Since $\rho\left(h^{-1}\right)$ is a group element in $X$, so $\rho(P) \rho(h)^{-1}$ is compact and open. Hence $\Phi$ is continuous.

2. $\Phi^{-1}$ is continuous: This can be proved by the same method as 1 .

(4.20) Proposition. Let $S$ be a wild second countable abelian $W$-semigroup. If the component of $S$ about $e$ is degenerate, then $S$ has a subsemigroup of Mostert type.

Proof. This follows from the fact that $P P^{-1}$ is such a wild subsemigroup of $S$.

\section{Local connectedness and connectedness.}

(5.1) Proposition. Suppose $S$ is a $W$-semigroup. If $G$ is locally connected under the induced topology, then $S$ is locally connected at each point of $G$.

Proof. Let $V$ be a neighborhood of $e$ such that $V \cap G$ is connected; then $(V \cap G) \subset V \subset(V \cap G)^{*}$. Since $G$ is dense in $S$, hence $V$ is connected. But such $V$ also forms a basis of neighborhoods of $e$ in $S$, and by the homogeneity of $G$, we have $S$ locally connected at each point of $G$.

(5.2) Corollary. If $G$ is locally connected, and $S$ is homogeneous in the sense that every two points have homeomorphic neighborhoods, then $S$ is locally connected and tame.

(5.3) Proposition. Suppose $S$ is a $W$-semigroup. If $G$ and $S-G$ both are locally connected under the induced topology, then $S$ is tame.

Proof. Suppose $S-G$ is dense. By the similar argument as in (5.1) $S$ is locally connected at each point of $S-G$. Thus $S$ is locally connected, a fortiori, $S$ is tame and $G$ is open. But this is impossible. Hence we know that $S-G$ is not dense in $S$. There is an open subset in $S$ which misses $S-G$. Hence $G$ is open in $S$ and $S$ is tame.

(5.4) EXAMPLES FOR (5.3). Under the assumptions of (5.3) we know that $S-G$ will not be dense. For example, let $S$ be the set of non-negative reals under the usual multiplication; then $S-G$ consists of only $\{0\}$. Let $S$ be the set of all the rationals with the form $1 / 2^{n}$ where $n$ can be any integer and $\{0\}$. In this example $S$ is not locally connected but $S-G$ is connected and locally connected, and $G$ is also locally connected.

(5.5) LEMMA. The component of identity $e$ in $G$ is a closed invariant subgroup of $G$.

Proof. The proof is similar to the case where $G$ is a topological group. We omit it.

(5.6) Proposition. Suppose $S$ is a $W$-semigroup. If $G$ is locally connected, 
then $S$ is tame if and only if the closure in $S$ of the component containing $e$ in $G$ is tame.

Proof. Let $K$ be the component of $e$ in $G$, and $K^{*}$ the closure of $K$ in $S$. The quotient group of $G$ over $K$ is totally disconnected, therefore $G / K$ is discrete since $G$ locally connected implies $G / K$ is also. It is clear that if $K$ is a topological group (i.e., if $K^{*}$ is tame), then $G$ is a topological group.

(5.7) Remark. The closure in $S$ of the component containing $e$ in $G$ might not be the same as the component of $e$ in $S$. For instance, let $R$ be the set of all real numbers under the ordinary multiplication; then the closure of the component of 1 in $R-\{0\}$ is the set of all non-negative numbers, and the component of 1 in $R$ is $R$.

(5.8) Proposition. Every second countable wild $W$-semigroup $S$ contains a subgroup which is a faithful representation of the real numbers, if the maximal dense subgroup $G$ is connected.

Proof. Assume $G$ has no subgroup which is a faithful representation of reals. Then the component $K(g)$ of the locally compact group $G(g)$ is compact [5]. Also, $K(g)$ is the invariant subgroup of $G$. Let $i$ be the inclusion map from $G(g)$ to $G, i$ is continuous, hence $i(K(g))=K$ is iseomorphic to $K(g)$.

Since $G(g) / K(g)$ is totally disconnected, there is an open subgroup $H(g)$ of $G(g)$ such that $H(g) / K(g)$ is compact. By the following analytic diagram,

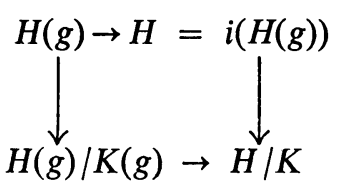

because $K(g)$ is compact and $H(g) / K(g)$ is compact, $H / K$ is compact also. Since $K$ is a compact subgroup of $G$, we form the decomposition space $S^{\prime}$ by defining $a \sim b$ if and only if $a K \cap b K \neq \Phi$. We notice that if $a K \cap b K \neq \square$, then $a K=b K$. Since the canonical map is open, we know $S^{\prime}$ is locally compact, second countable and hence metrizable. Thus $G^{\prime}=G / H$ is metrizable and connected, and its cardinality must be the same as the continuum if it is not degenerate. But $G^{\prime \prime}=G(g) / H(g)$ is discrete, second countable, and hence $G^{\prime \prime}$ must be at most countable. This implies that $G^{\prime}$ is degenerate. Thus $G=H$, and hence $G$ is a compact topological group. From this contradiction we know that $G$ must contain a faithful representation of the reals.

(5.9) Proposition. If $S$ is a $W$-semigroup and $G$ is connected, then for any neighborhood $V$ of $e$, the closure of $\bigcup_{n>0} V^{n}=S$.

Proof. Let $A=\bigcup_{n>0} V^{n}$; then $A$ is a semigroup, hence $A^{*}=$ closure of $A$ in $S$ is a semigroup. If $g \in A^{*} \cap G$, then $g \in g V \cap G \subset A^{*} \cap G$. Hence $A^{*} \cap G$ 
is open in $G$. Also $A^{*} \cap G$ is closed in $G$. Because $G$ is connected, this implies $G \subset A^{*}$. But $G^{*} \subset A^{*} \subset S=G^{*}$. Hence $S=$ closure of $A=$ closure of $\bigcup_{n>0} V^{n}$.

(5.10) Proposition. Let $S$ be a $W$-semigroup. If $S-G$ is connected, $G$ is second category and $S$ is fully normal. Then $S$ is tame.

Proof. If $S-G$ is dense in $S$, then $S$ is connected. By Proposition (6.4), $S$ is tame. But if $S$ is tame, then $G$ is open and $S-G$ cannot be dense in $S$. If $S-G$ is not dense, then there is an open set $V$ which misses $S-G$, a fortiori, $V G=G$ is open in $S$. This implies $S$ is tame.

(5.11) Proposition. Let $K_{a}$ denote the component in $S$ which contains $a \in S$. Then $K_{g}$ is homeomorphic to $K_{f}$ for any two elements $g, f \in G$.

Proof. We are going to prove that $K_{g}$ is homeomorphic to $K_{e}$ for any $g$ in $G$. Define $\Phi_{g}: K_{e} \rightarrow K_{g}$ by $\Phi_{g}(k)=g k$. Since $g K_{e} \cap K_{g} \neq 0, g K_{e} \subset K_{g}$. It is clear that $\Phi_{g}$ is a homeomorphism. Hence we only need to prove that it is an onto map. Since $g^{-1} K_{g} \cap K_{e} \neq 0, g^{-1} K_{g} \subset K_{e}$. Thus we know for any $s \in K_{g}, \Phi_{g}\left(g^{-1} s\right)$ $=g g^{-1} s=s$.

\section{Second category.}

(6.1) Proposition. If $G$ is second category and $H$ is a closed subgroup of $G$, then the left coset space $G^{\prime}=G / H$ is also second category.

Proof. Suppose $G^{\prime}$ is not second category. Then $G^{\prime}=\bigcup_{i} A_{i}$, where $A_{i}^{*}=A_{i}$, interior of $A_{i}=\square$ and $A_{i} \subset G^{\prime}$. Let $\pi$ be the canonical map from $G$ to $G^{\prime}$. Then $\pi^{-1}\left(A_{i}\right)$ is closed and $\pi^{-1}\left(A_{i}\right)$ has an empty interior, since if $\pi^{-1}\left(A_{i}\right)$ has nonvoid interior, then $A_{i}=\pi\left(\pi^{-1}\left(A_{i}\right)\right)$ has nonvoid interior also. This is a contradiction. Hence $G^{\prime}$ is second category.

(6.2) LeMmA. If $S$ is a $\sigma$-compact semigroup, then any second category subgroup $H$ of $S$ is a topological group.

Proof. Since $S$ is $\sigma$-compact, $H \subset \bigcup_{i} K_{i}$ where $K_{i}$ is a compact subset of $S$. Also $H \subset \bigcup_{i} K_{i}^{-1}$, and $K_{i}^{-1}$ is closed. Since $H$ is second category and each $K_{i}^{-1} \cap H$ is closed in $H$, there is a set $K_{i}$ such that $K_{i}^{-1} \cap H$ has nonvoid interior $V_{0}$ in $H$. Let $h \in V_{0}$, then $A=h^{-1}\left(K_{i}^{-1} \cap H\right)$ is an open neighborhood of $e$ in $H$. It is clear that if any net $a_{v} \in A$ converges to $e$, then $a_{v}^{-1}$ converges to $e$ also. Hence $H$ is a topological group.

(6.3) LeMmA. If $S$ is a $W$-semigroup and $S$ is a Lindelöf space, then $S$ is tame if and only if $G$ is second category.

Proof. If $S$ is locally compact and Lindelöf, then $S$ is $\sigma$-compact. If $G$ is second category, then by (6.2) $G$ is a topological group.

(6.4) Proposition. If $S$ is a connected $W$-semigroup and $S$ is fully normal, then $S$ is tame if and only if $G$ is second category. 
Proof. If $S$ is connected locally compact and fully normal, then $S$ is $\sigma$-compact. Thus the assertion of the theorem follows from (6.3).

(6.5) Proposition [9]. Suppose G is a second countable and second category regular space. If $G$ is a group and its group multiplication is continuous separately, then $G$ is a topological group under the group operation.

\section{Miscellaneous topics.}

(7.1) Definition. A Hausdorff Baire space $X$ is a weak T-space if and only if for every one-one function $h$ from $X$ to $X$, if the image of $X$ under $h$ is dense in $X$, and if $h$ is a continuous limit of a net of homeomorphisms from $X$ onto $X$, then $h(X)=X$.

(7.2) Definition (Mostert [8]). A locally compact space $X$ is a $T$-space if and only if for every one-one function $h$ from $X$ to $X$, if the image of $X$ under $h$ is dense in $X$, and if $h$ is a continuous limit of a net of homeomorphisms from $X$ onto $X$, then $h$ is a homeomorphism from $X$ onto $X$.

(7.3) Proposition. A T-space is also a locally compact weak T-space.

Proof. Let $X$ be a $T$-space and let $h$ be the function which satisfies all the assumptions in (7.2). Then $h$ is a homeomorphism from $X$ onto $X$. Certainly this implies that $h$ is an onto map.

(7.4) Lemma. If $S$ is a semigroup with a dense subgroup $G$, if its topology is first countable and if it is a weak T-space, then $G$ is second category.

Proof. Let $R=\left(\bigcap_{n} V_{n} G\right) \cap\left(\bigcap_{n} G V_{n}\right)$, where $\left\{V_{n} \mid n \in \omega\right\}$ is a countable neighborhood basis at $e$. Since $G$ is dense, $V_{n} G$ and $G V_{n}$ are open dense subsets of $S$, therefore $R$ is second category. Now, we will prove that $G=R$. Let $f \in R$. Then $f=v_{n} g_{n}=g_{n}^{\prime} v_{n}^{\prime}, n=1,2, \cdots$, and $v_{n}, v_{n}^{\prime} \in V_{n}, g_{n}, g_{n}^{\prime} \in G$. If $f s=f t$, then $g_{n}^{\prime} v_{n}^{\prime} s=g_{n}^{\prime} v_{n}^{\prime} t$. Hence $v_{n}^{\prime} s=v_{n}^{\prime} t$ for $n=1,2,3, \cdots$, which implies $\lim v_{n}^{\prime} s=\lim v_{n}^{\prime} t$. Since $\lim _{n} v_{n}^{\prime}=e, s=t$, so that $s \rightarrow f s$ is a one-one map.

To see that $f S$ is dense in $S$, let $V$ be any open subset of $S$. Then there is an element $g \in G$ and $g \in V$. Because $e g=g e=g \in V$, there exists an integer $p$ such that $V_{p} g \subset V$. Let $f=v_{p} g_{p}$, then $f g_{p}^{-1} g=v_{p} g_{p} g_{p}^{-1} g=v_{p} g \in V_{p} g \subset V$. Hence $f S \cap V \neq \square$, and $f S$ is dense in $S$.

Since $G$ is dense in $S, f$ is the continuous limit of a net of elements in $G$. By assumption $S$ is a weak $T$-space; therefore we have $f S=S$. By a dual argument, $S f=S$, which implies $f \in G$. Hence $G=R$ is second category.

(7.5) Proposition. If $S$ is a semigroup with a dense subgroup $G$, and if $S$ is a second countable weak $T$-space, then $S$ is tame.

(7.6) Proposition. If $S$ is a first countable $W$-semigroup, if $S-G$ is connected, and $S$ is a weak $T$-space, then $S$ is tame. 
(7.7) $A$ weak $T$-space may not be a T-space. Let $X$ be the subset $\left\{2^{n} \mid-\infty \leqq n<\infty\right\}$ of the real line. Define $h_{l}$ as follows:

1. $h_{l}\left(2^{2 K}\right)=2^{-2 k}, 0 \leqq k \leqq l$,

2. $h_{l}$ maps the set $\left\{2^{n} \mid n \geqq 0, n \neq 2 i, 0 \leqq i \leqq k\right\}$ one-to-one and onto the set $\left\{2^{n} \mid n \geqq 0\right\}$ following the linear order of each set.

3. $h_{l}$ onto the set $\left\{2^{n} \mid n<0\right\}$ one-one and onto the set $\left\{2^{n} \mid n<0, n \neq 2 i\right.$, $-k \leqq i \leqq 0\}$ by the linear order.

4. $h_{l}(0)=0$. It is clear that $h=\lim h_{v}$ is one-one and onto, but $h^{-1}$ is not continuous since $2^{-2 n}$ converges to 0 when $n>0$. Since $h^{-1}\left(2^{-2 n}\right)=2^{2 n}$ has no limiting point, we know $X$ is not a $T$-space; however, $X$ is a weak $T$-space. Because there is only one limiting point 0 , the rest of the points are isolated points. If $h(X)$ is dense in $X, h(X) \supset X-\{0\}$. Now $h(0)$ has to be 0 because the image of the limiting point has to be the limiting point of $h(X)$. So we have $h(X)=X$. Mostert raised the question: Is a locally compact space a $T$-space? Later on he shows that the answer is negative by the wild semigroup of Mostert type. The above space is another such example.

(7.8) It would be interesting to find out what kind of spaces are $T$-spaces. It is clear that compact spaces or locally compact pseudo-compact spaces are $T$-spaces.

(7.9) Let $X$ be a locally compact semigroup, and $\tilde{X}$ its one point compactification, obtained by adjoining the point $\omega$. If we define the multiplication of $\tilde{X}$ by $\omega \cdot x=\omega=\omega \cdot x$, then $\tilde{X}$ is an algebraic semigroup, but multiplication is not, in general, continuous.

(7.10) Proposition. If $S$ is a $W$-semigroup and $\tilde{S}$ is the one point compactification of $S$ as in (7.9), then $\tilde{S}$ is a topological semigroup if and only if $G=S$ and $S$ is compact.

Proof. If $\tilde{S}$ is a topological semigroup, then $\tilde{S}$ is a compact semigroup. Hence $G$ is a topological group and therefore $G=G^{*}=S$.

(7.11) Definition. Let $S$ be a semigroup and $\tilde{S}=S \cup\{\omega\}$, the one point compactification of $S$. $S$ is called a Hofmann semigroup if there is an element $s \in S$ such that the multiplication in $\tilde{S}$ is jointly continuous at $(s, \omega)$.

(7.12) Proposition. AW-semigroup $S$ is tame if and only if $S$ is a Hofmann semigroup.

Proof. 1. Sufficiency. Suppose $S$ is a Hofmann semigroup. Then the multiplication in $\tilde{S}$ is continuous at some point, say $(s, \omega)$, so we can find a compact neighborhood $V_{s}$, and a compact subset $V$ in $S$ such that $V_{s}(S-V) \subset S-\{e\}$. Since $G$ is dense in $S$, there is $g \in G$ such that $g \in V_{s}$. Let $\left\{g_{v}\right\}$ be a set in $G$ such that $\lim g_{v}=e$. Now $g \lim g_{v}=\lim g g_{v}=g \in V_{s}, g g_{v}(S-V) \subset S-\{e\}$ for most 
$g_{v}$. Since $g g_{v}(S-V)=S-g g_{v} V \subset S-\{e\}, g g_{v} V \supset\{e\}, g V \ni g_{v}^{-1}$ and $g V$ is compact, then $\left\{g_{v}^{-1}\right\}$ must converge to some point; a fortiori, the limit point of $\left\{g_{v}^{-1}\right\}$ is $e$. This shows that $G$ is a topological group.

2. Necessity. Suppose $S$ is tame, i.e., $G$ is a topological group. Then the $g$-topology on $G$ is the same as $k$-topology. Let $K$ be a compact subset of $S$ and let $U$ be an open subset of $S$ containing $K$ with compact closure, $K \subset U \subset U^{*} \subset S$. Then $e \in[S|U, S| K],[S|U, S| K]$ is a neighborhood of $e$, so for $g \in[S|U, S| K]$, $g(S \mid U) \subset S|K, S| g U \subset S \mid K$, a fortiori, $[S|U, S| K](S \mid U) \subset S \mid K$. This shows that multiplication is continuous at $(e, \omega) . S$ is a Hofmann semigroup.

(7.13) REMARK. The structure of the Hofmann semigroup with compact boundary (i.e., $S \mid G$ is compact) has been solved by K. L. Hofmann. For a definition of the Hofmann semigroup slightly different from ours, see P. S. Mostert [8].

(7.14) Very little is known about the homomorphisms of $W$-semigroups. The following is a trivial example which shows the existence of homomorphism from a wild semigroup onto a tame $W$-semigroup. Let $S$ be any wild $W$-semigroup and $T$ a locally compact semigroup. The cartesian product $X$ of $S$ and $T$ is a wild $W$-semigroup. Let $p$ be the projection from $X$ onto $T$. It is clear that $p$ is an onto homomorphism. We do not know if there is a homomorphism from a tame semigroup onto a wild semigroup or not. The following are a few observations along this line. For completeness we quote a theorem by Mostert.

(7.15) Proposition [8]. Assume $S$ and $S^{\prime}$ are second countable $W$-semigroups with maximal dense subgroups $G$ and $G^{\prime}$ respectively. If there is an onto homomorphism $h: S \rightarrow S^{\prime}$ such that $h^{-1}\left(G^{\prime}\right)=G$, then either $S$ and $S^{\prime}$ are tame or both are wild.

(7.16) Proposition. Let $h$ be a homomorphism from $S$ to $S^{\prime}$, where $S$ and $S^{\prime}$ are $W$-semigroups and $S$ is tame. Then there is a homomorphism $\tilde{h}: G \rightarrow G^{\prime}(g)$ such that $i \tilde{h}=h, i$ is the canonical map from $G^{\prime}(g)$ onto $G^{\prime}(k) \subset S^{\prime}$.

Proof. We understand that $G(g)$ is obtained from $G$ by retopologizing $G(k)$, so there is a natural one-one continuous onto map: $G^{\prime}(g) \rightarrow G^{\prime}(k)$. Define $\tilde{h}(f)=i^{-1} h(f), f \in G$. Since $\tilde{h}\left(f f^{\prime}\right)=i^{-1} h\left(f f^{\prime}\right)=i^{-1}\left(h(f) h\left(f^{\prime}\right)\right)$ $=\left(i^{-1} h(f)\right)\left(i^{-1} h(f)\right)\left(i^{-1} h\left(f^{\prime}\right)\right)=\tilde{h}(f) \tilde{h}\left(f^{\prime}\right), \tilde{h}$ is a homomorphism. Suppose $\left\{f_{v}\right\} \in G$ is a set converging to $e, G$ is a topological group, and $\left\{f_{v}^{-1}\right\}$ converges to $e$. Then $\left\{h\left(f_{v}\right)\right\}$ and $\left\{h\left(f_{v}^{-1}\right)\right\}$ both converge to $e^{\prime}$. It is known that if $\left\{h\left(f_{v}\right)\right\}$ and $\left\{h\left(f_{v}\right)\right\}^{-1}$ both converge to $e^{\prime}$ in $G(k)$, then $\left\{h\left(f_{v}\right)\right\}$ as a set in $G(g)$, i.e., $\left\{i^{-1} h\left(f_{v}\right)\right\}$ also converges to $e^{\prime} \in G(g)$. This shows that $\tilde{h}$ is continuous at identity. The rest of the proof of the continuity follows under the same pattern.

(7.17) Remark. Since so far the only wild semigroups known to us essentially are semigroups of Mostert type, let us observe the onto homomorphism (if such 
exists) from a tame $W$-semigroup to a semigroup $S^{\prime}$ of Mostert type. Since the $G^{\prime}(g)$ is discrete, from (7.16) the kernel of $\tilde{h}$ has to be open and closed. In other words, the quotient $G / \operatorname{kernel} \tilde{h}$ is discrete too. This seems to support the following conjecture: there does not exist a homomorphism from a tame semigroup onto a semigroup of Mostert type.

(7.18) Proposition. If $S$ and $S^{\prime}$ are $W$-semigroups, $S$ is tame. If $h$ is a homomorphism from $S$ onto $S^{\prime}$ such that there is $s \in S \mid G, h(s)=g^{\prime} \in G^{\prime} \subset S^{\prime}$. Then $h(S \mid G)=S^{\prime}$.

Proof. Since $h(s)=g^{\prime}$ there is $t \in S, h(t)=g^{\prime-1}, h(s t)=e^{\prime}$. It is clear that $s t \notin G$, otherwise $s \in G$, contradicts that $s \in S \mid G$. Let $f^{\prime} \in G^{\prime}$. Since $h$ is onto, there is $p \in S$ such that $h(p)=f^{\prime}$. If $p \in G$, then $\operatorname{st} p \in S \mid G, h(s t p)=e^{\prime} f^{\prime}=f^{\prime}$, so we know $h(S \mid G) \supset G^{\prime}$. Now assume $g \in G, h(g)=s^{\prime} \in S^{\prime} \mid G^{\prime}$. Then $h\left(g g^{-1}\right)=h(g) h\left(g^{-1}\right)=s^{\prime} h\left(g^{-1}\right)=e^{\prime}$. This implies $h(g)=s^{\prime} \in G^{\prime}$. So $h(G) \cap\left(S^{\prime} \mid G^{\prime}\right)=\Phi$, a fortiori, $h(S \mid G)=S^{\prime}$.

Added in proof. Professor R. W. Bagley had kindly informed us that the proof of the theorem in [9] had a gap. However, the statement of the theorem is correct and the original proof can be fixed by using a slightly weak form of M. K. Fort's category theorem [M. K. Fort, Jr., Fund. Math. 42 (1955), 277-288].

\section{REFERENCES}

1. R. Arens, Topologies for homeomorphism groups, Amer. J. Math. 68 (1946), 593-610.

2. R. Ellis, Locally compact transformation groups, Duke Math. J. 24 (1957), 119-125.

3. - - A note on the continuity of the inverse, Proc. Amer. Math. Soc. 8 (1957), 372-373.

4. K. H. Hofmann, Lokalkompakte auhsammenhangende topologische Halbgruppen mit dichter Untergruppe, Math. Ann. 140 (1960), 22-32.

5. D. Montgomery and L.Zippin, Topological transformation groups, Interscience, New York. La., 1953.

6. R. J. Koch, On topological semigroups, Dissertation. Tulane Univ., New Orleans,

7. A. D. Wallace, The structure of topological semigroups, Bull. Amer. Math. Soc. 61 (1955), 95-112.

8. P. S. Mostert, Untergruppen von Halbgruppen, Math. Z. 82 (1963), 29-36.

9. T. S. Wu, Continuity in topological groups, Proc. Amer. Math. Soc. 13 (1962), 452-453.

10. B. Eckmann, Uber monothetische Gruppen, Comment. Math. Helv. 16 (1943/1944), 249-263.

11. E. Hewitt, Compact monothetic semigroups, Duke Math. J. 23 (1956), 447-457.

TULANE UNIVERSITY,

New OrLeans, Louisiana

STANFORD UNIVERSITY,

StANFord, CALIFORNiA 